Draft version December 5, 2018

Preprint typeset using $\mathrm{LAT}_{\mathrm{E} X} \mathrm{X}$ style emulateapj v. 12/16/11

\title{
EXPECTED IMPRINTS OF THE CAROUSEL IN MULTI-FREQUENCY PULSAR OBSERVATIONS AND NEW EVIDENCE FOR MULTI-ALTITUDE EMISSION
}

\author{
YOGESH MAAN \\ ASTRON, Netherlands Institute for Radio Astronomy, Oude Hoogeveensedijk 4, 7991 PD, Dwingeloo, The Netherlands \\ Draft version December 5, 2018
}

\begin{abstract}
Subpulse modulation has been regarded as one of the most insightful and intriguing aspects of pulsar radio emission. This phenomenon is generally explained by the presence of a carousel of sparks in the polar acceleration gap region that rotates around the magnetic axis due to the $\mathrm{E} \times \mathrm{B}$ drift. While there have been extensive single pulse studies, geometric signatures of the underlying carousel, or lack thereof, in simultaneous multi-frequency observations have remained largely unexplored. This work presents a theoretical account of such expected signatures, particularly that of a geometry induced phase-offset in subpulse modulation, including various formulae that can be readily applied to observations. A notable result is a method to resolve aliasing in the measured subpulse modulation period without relying on knowledge of the viewing geometry parameters. It is also shown in detail that the geometry induced phase-offset enables critical tests of various observed phenomena as well as proposed hypotheses, e.g., multi-altitude emission, magnetic field twisting, pseudo-nulls, etc., in addition to that of the carousel model itself. Finally, a detailed analysis of a $327 \mathrm{MHz}$ pulse-sequence of PSR B1237+25 is presented as a case study to test the single-frequency multi-altitude emission scenario. The analysis provides a firm evidence of inner and outer conal components of this pulsar to have originated from the same carousel of subbeams and emitted at different heights.
\end{abstract}

Keywords: Pulsars: general, pulsars: individual $(\mathrm{B} 1237+25)$, radiation mechanisms: non-thermal, stars: neutron

\section{INTRODUCTION}

A considerable fraction of pulsars exhibit systematic variation in position and intensity of single pulse components when viewed from one pulse to other. The single pulse components are called subpulses, and a systematic variation in their position make them appear to drift in pulsar's rotation phase. This fascinating phenomenon is known as subpulse drifting (Drake \& Craft 1968), and has been regarded as one of the most insightful aspects of the pulsar radio emission. The phenomenon is generally understood in the context of the widely adopted "carousel model" (Ruderman \& Sutherland 1975). This model attributes subpulse drifting to the presence of a carousel of regularly spaced 'sparks' circulating around the magnetic axis in the polar cap region of the star because of the $\mathrm{E} \times \mathrm{B}$ drift. The sparks give rise to plasma beams streaming upward from the surface along the magnetic field lines. At different altitudes, these "subbeams" emit radiations in different radio frequency ranges. A single carousel could give rise to subpulse modulation in a maximum of two pulse components. To explain modulation in more than two components observed for several pulsars, Gil \& Sendyk (2000) proposed a maximal packing of the sparks and effectively presence of more than one carousel on the polar cap. More recently, it has been suggested that the $\mathrm{E} \times \mathrm{B}$ drift of the sparks is around the point of maximum potential at the polar cap van Leeuwen \& Timokhin 2012; Szary \& van Leeuwen 2017) which could help in explaining some other observed modulation varieties such as bi-drifting.

The subpulse modulation properties are important observables that enable probes of the plasma configurations

Electronic address: maan@astron.nl in the magnetosphere as well as help in examining various physical models. For example, coherent subpulse modulations in single pulse sequences of a few pulsars have been used to map the accessible parts of the subbeam configurations (e.g., Deshpande \& Rankin 1999, 2001; Mitra \& Rankin 2008; Maan \& Deshpande 2014). The expected subpulse modulation phase, for a given pulse longitude, depends on the viewing geometry as well as the number of sparks in the carousel. This relationship enables constraining the circulation period of the carousel for a known emission geometry. The modulation phase envelope across the pulse-profile has also been used to scrutinize the carousel model or its subsequent variants (e.g., Edwards et al. 2003; Maan \& Deshpande 2014).

Several of the observed pulsar properties depend on the altitude where the radio emission originates. For example, the pulsar radio emission beam width, and hence the observed pulse-width becomes larger at low frequencies due to the dipolar flaring of the field lines (Cordes 1978). The position angle of the linearly polarized intensity also depends on the magnetic configuration at the emission site. Depending on the emission altitude, the average total intensity and polarization position angle profiles also show effects of aberration and retardation (Blaskiewicz et al. 1991). However, the subpulse modulation phase is primarily decided by the circulation of the carousel at the polar cap and how the plasma subbeams propagate to the emission sites. The foot-points of the magnetic field lines that give rise to the observed emission at different altitudes differ slightly in their magnetic colatitudes at the polar cap. Hence, the observed emission at different altitudes samples slightly different parts of the seed spark pattern at the polar cap at its 
different rotation phases. This work presents the implications of this aspect in multi-frequency and wide-band observations of subpulse modulations in pulsars ${ }^{1}$. The preliminary results of this work were presented in the conference "Pulsar Astrophysics - The Next 50 Years" (Maan 2018).

Section 2 provides a detailed physical description and formulation of the frequency-dependent subpulse modulation phase-offsets that are expected to be observed for a given viewing geometry and demonstrates the effect using simulations. This section also presents methods to utilize the above expected phase-offsets to determine several physical parameters related to the carousel. The frequency-dependent phase-offsets also enable tests of several observed or proposed phenomena that are often discussed within the scope of the carousel model. Some of these tests are briefly described in Section 3 . Section 4 presents a practical application of this aspect using a sensitive single pulse sequence of PSR B1237+25 and provides firm evidence for single frequency multialtitude emission in this pulsar. The overall conclusions are presented in Section 5.

\section{FREQUENCY-DEPENDENT SUBPULSE MODULATION PHASE-OFFSET: FORMULATION AND DEMONSTRATION}

Radius-to-frequency mapping (hereafter RFM; Cordes 1978) suggests that the radio signals at different frequencies originate at different altitudes from the pulsar's surface, with lower frequency signals being emitted farther away. Furthermore, for a given viewing geometry, the observed emission originates from a set of field lines that are parallel to the observer's line-of-sight at the emission altitude (see Figure 1(a). This aspect, along with the dipolar configuration of the magnetic field lines and RFM, implies that the observed emission at different frequencies originates from slightly different sets of fields lines, with the lower frequency emission originating from (magnetic) poleward field lines (see, for example, Deshpande \& Rankin 2001; Edwards \& Stappers 2003; Maan et al. 2013). Hence, the observed radio emission in different frequency ranges sample slightly different parts of the polar-cap and active plasma processes therein, e.g., the carousel of sparks (see Figure 1(a)). Since the observed subpulse modulation results from a set of emission sub-beams that stem from, and co-rotate with, a carousel of sparks on the polar-cap, observations at different frequencies would sample the carousel at its different rotation phases.

Figure 1(b) presents a simulated carousel of sparks on the polar cap along with the footpoint-traverses of field lines responsible for emission at two different frequencies for a given viewing geometry. The assumed frequencies are 240 and $610 \mathrm{MHz}$, and the corresponding emission altitudes are estimated to be about 710 and $560 \mathrm{~km}$, respectively, using the empirical formula given by Kijak \& Gil (2003, see their equation 3 ). The viewing geometry parameters (magnetic inclination angle, $\alpha=50^{\circ}$, and im-

\footnotetext{
${ }^{1}$ During manuscript preparation (cf. Maan 2018), a similar description was presented by Bilous (2018) for PSR B0943+10. Our independent formulation presented in Section 2.1 introduces a minor improvement in the expression given by Bilous (2018), and also demonstrates how the phase-offset depends on other pulsar quantities.
}

pact angle $\beta=2.6^{\circ}$ ) and the rotation parameters (period, $P=1.28 \mathrm{~s}$, and period-derivative, $\dot{P}=6.8 \mathrm{e}-15)$ are assumed to be those known for the bright radio pulsar PSR B0834+06. From the two emission heights, the magnetic field lines corresponding to the sightline traverse are traced back up to the pulsar surface to determine the corresponding footpoint traverse. As expected, the lower frequency emission samples poleward part of the polar cap. This fact has been exploited to map larger fraction of the underlying emission patterns utilizing the circulation of the carousel (e.g., Deshpande \& Rankin 2001; Asgekar \& Deshpande 2001; Maan et al. 2013). The peaks of the pulse components correspond to field lines which have their footpoints on the "circumference" of the carousel defined by the peaks of the individual sparks. The resultant average profile will naturally have two components (similar to those in the bottom panels of Figure 3). As apparent from Figure 1(b), simultaneous observations of subpulse modulation in the corresponding pulse-components at two frequencies would exhibit a phase-offset. Moreover, for a given circulation direction of the carousel, the observed phase-offset in subpulse modulations at two frequencies in the leading and trailing components will be equal in magnitude but opposite in sign. Without loss of generality and for ease of description, unless stated otherwise, discussion in the rest of the paper would assume a viewing geometry similar to that shown in Figure 1(b) resulting in two pulse components.

\subsection{Formulation}

The subpulse modulation phase, $\Theta$, as a function of pulse longitude consists of three terms: (1) a scaled version of the magnetic azimuth, where the scaling factor is the number of uniformly distributed sparks, $N_{\mathrm{sp}}$, in the carousel, (2) an additional, pulse longitude dependent phase term which accounts for the carousel rotation over the course of the pulse, and (3) a reference modulation phase at a reference pulse longitude. The first term also includes the sign of the modulation phase gradient which depends on the relative direction of the pulsar rotation and the carousel circulation and is given by sign of $\beta$. For a known viewing geometry, the magnetic azimuth corresponding to a pulse phase $\phi$ is given by:

$$
\chi(\phi)=\arctan \left(\frac{\sin \zeta \sin \left(\phi-\phi_{0}\right)}{\sin \alpha \cos \zeta-\cos \alpha \sin \zeta \cos \left(\phi-\phi_{0}\right)}\right)
$$

where, $\zeta=\alpha+\beta$, and $\phi_{0}$ is the pulse longitude that corresponds to the fiducial plane containing the magnetic and rotation axes. Due to the aberration effects, the conal emission shifts forward by $r_{\nu} / \mathrm{R}_{\mathrm{LC}}$, where $r_{\nu}$ is the emission altitude and $\mathrm{R}_{\mathrm{LC}}$ is the light cylinder radius (Gupta \& Gangadhara 2003; Dyks et al. 2004). On the other hand, the core-emission shifts backward by a similar amount due to retardation effects. In rest of the paper, $\phi_{0}$ has been set to zero assuming that its location is correctly identified after correcting for the aberration/retardation effects. Following Edwards \& Stappers (2002), the subpulse modulation phase is then given by:

$$
\Theta(\phi)=-N_{\mathrm{sp}} \operatorname{sgn} \beta \chi(\phi)+\left(n+\frac{P}{P_{3}^{\mathrm{obs}}}\right) \phi+\Theta_{0}
$$




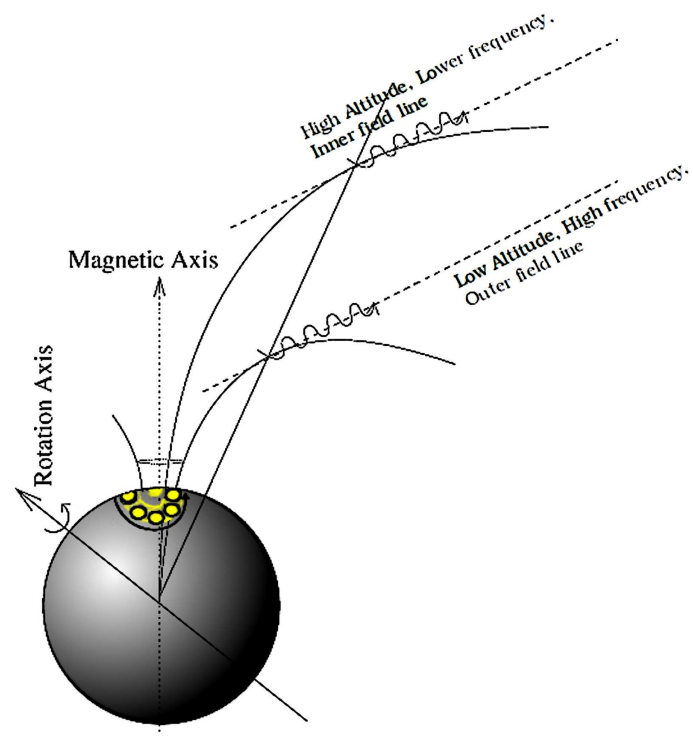

(a)

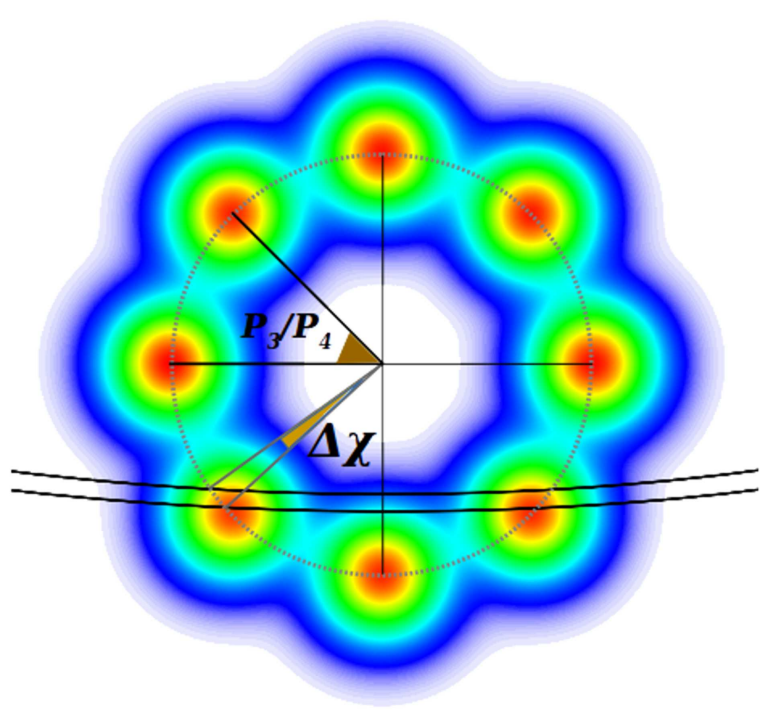

(b)

Figure 1. Left: A demonstration of radius-to-frequency mapping of radio emission from pulsars. The figure is not up-to-scale, primarily to show that the high frequency emission originates at a lower altitude and from slightly outer field lines when compared to the low frequency emission. Right: A simulated carousel of sparks and sightline traverses corresponding to 240 and $610 \mathrm{MHz}$ traced back to the pulsar surface. The inner (poleward) traverse correspond to the lower frequency. It is clearly evident that the two frequencies would sample the carousel at its different rotation phases. The instantaneous offset in magnetic azimuth is denoted by $\Delta \chi$. The angular separation of consecutive sparks is proportional to the ratio of the subpulse modulation and the carousel circulation periods $\left(\mathrm{P}_{3} / \mathrm{P}_{4}\right)$.

where, $P_{3}^{\text {obs }}$ and $n$ are the observed primary modulation period and the aliasing order, respectively, and $\Theta_{0}$ is the modulation phase at $\phi_{0}$. The actual modulation period, $P_{3}$, can be expressed as $P / P_{3}=n+P / P_{3}^{\text {obs }}$, and $-0.5<$ $P / P_{3}^{\text {obs }}<0.5$. The circulation period of the carousel is then $P_{4}=N_{\mathrm{sp}} \times P_{3}$.

Assuming the magnetic field to be of dipolar configuration, the magnetic azimuth for any given field line is a constant. In other words, the magnetic azimuth for a given pulse-longitude is independent of the emission altitude. Hence, the modulation phase-offset at the peak of a given pulse-component resulting from simultaneous observations at a pair of frequencies is entirely decided by the difference in the corresponding pulse-longitudes and the corresponding change in the magnetic azimuth. For aberration-corrected pulse-longitudes of the peaks of a certain component at two frequencies, $\phi_{1, \nu_{1}}, \phi_{1, \nu_{2}}$, the expected subpulse modulation phase-offset is:

$$
\Delta \Theta=-N_{\mathrm{sp}} \operatorname{sgn} \beta \Delta \chi+\left(n+\frac{P}{P_{3}^{\text {obs }}}\right) \Delta \phi
$$

where $\Delta \chi=\chi\left(\phi_{1, \nu_{1}}\right)-\chi\left(\phi_{1, \nu_{2}}\right)$, and $\Delta \phi=\phi_{1, \nu_{1}}-\phi_{1, \nu_{2}}$. It is worth mentioning here that $\Delta \Theta$ is essentially the difference between subpulse modulation phases at two different pulse-longitudes, but observed almost simultaneously at two different frequencies. The above expression correctly describes the difference in modulation phase for given pulse longitudes at a given emission height. However, to account for the emission at different altitudes and to compensate for the carousel rotation during the corresponding light travel time, ideally an additional term, $\delta \Theta$, needs to be introduced:

$$
\Delta \Theta=-N_{\text {sp }} \operatorname{sgn} \beta \Delta \chi+\left(n+\frac{P}{P_{3}^{\text {obs }}}\right) \Delta \phi+\delta \Theta
$$

Denoting the emission altitudes at the two frequencies to be $r_{\nu_{1}}$ and $r_{\nu_{2}}, \delta \Theta$ can be expressed as:

$$
\delta \Theta=\left(n+\frac{P}{P_{3}^{\text {obs }}}\right) \frac{2 \pi\left(r_{\nu_{1}}-r_{\nu_{2}}\right)}{c P}
$$

For difference of a few hundred kilometers in the emission altitudes, the light travel time would be only of the order of a millisecond. For a typical $P_{3}$ of the order of seconds, $\delta \Theta$ would be negligible in majority of the cases.

The second term in equation 3 primarily depends on $\Delta \phi$ and the aliasing order. To estimate the typical expected values of $\Delta \phi$, we use the geometrical expression for the opening angle of the emission cone (Lorimer, \& Kramer 2004): $\rho=$ $1.24^{\circ}\left(r_{\nu} / 10 \mathrm{~km}\right)^{0.5}(P / s)^{-0.5}$. For $P=1.28 \mathrm{~s}$ and emission heights of 710 and $560 \mathrm{~km}$ corresponding to the assumed frequencies of 240 and $610 \mathrm{MHz}$, the opening angles turn out to be $9.2^{\circ}$ and $8.1^{\circ}$, respectively. The pulse-longitudes where the sightline cuts with the cone boundaries on the left side of the fiducial plane at the two emission heights are assumed to be the peak positions of the corresponding pulse components, i.e., $\phi_{1, \nu_{1}}$ and $\phi_{1, \nu_{2}}$. For $-6.5^{\circ}<\beta<+6.5^{\circ}, 10^{\circ}<\alpha<170^{\circ}$, the left panel in Figure 2 shows the variations of $\Delta \phi$. For $\alpha$ between about $20^{\circ}$ and $160^{\circ}, \Delta \phi$ is only a few degrees. For such viewing geometries and the assumed emission heights, only very high aliasing orders $(|n| \gtrsim 5)$ would result in the second term to be at a observationally measurable level. For smaller inclination angles, i.e., nearly 


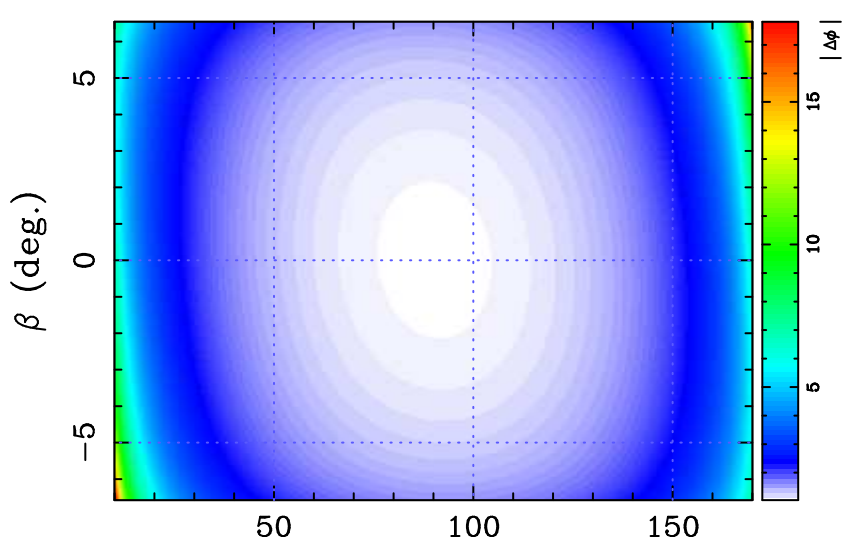

$\alpha($ deg.)

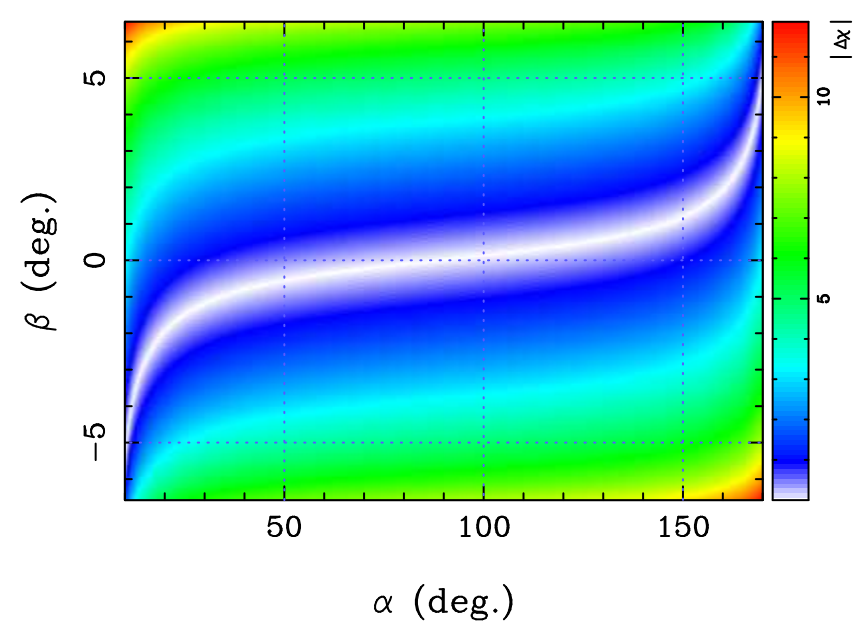

Figure 2. Left: Expected change in longitude position (in deg.) of a pulse-component observed from emission heights 710 and 560 km (see text for other details), as a function of $\alpha$ and $\beta$. Right: The corresponding absolute variations in the magnetic azimuth (i.e., $|\Delta \chi|$; in deg.), as a function of the viewing geometry parameters.

aligned rotators, $\Delta \phi$ could be $10-15^{\circ}$ or even more. For such cases, the contribution of the second term could be significant even for first aliasing order.

For a reasonably large number of sparks, the first term in equation 3 is expected to have dominant contribution in majority of the cases. For $\Delta \phi$ values in the left panel of Figure 2, the right panel shows the corresponding absolute changes in the magnetic azimuth (i.e., $|\Delta \chi|)$. The white colored region in this panel $(\Delta \chi=0)$ suggests that the points where the sightline cuts the cone boundaries at the two emission heights lie on a magnetic radial line. For such viewing geometries, the contribution of the first term will naturally be zero irrespective of the number of sparks. For the nearby viewing geometries where $\Delta \chi$ is only a few degrees, a large number of sparks would help. For all other regions, $\Delta \Theta$ should be easily noticeable even for a few sparks in the carousel.

Figure 2 provides an estimate of the expected $\Delta \phi$ and $\Delta \chi$ with the underlying assumptions, however, the actual quantities would differ depending on the observing frequencies and the actual emission cone size. For example, $\Delta \chi$ would vary more steeply with $\beta$ for narrower cones.

Note that the observed $\Delta \Theta$ can also be exploited to constrain the viewing geometry, especially if $N_{\mathrm{sp}}$ is known independently. Particularly for small inclination angles where $\Delta \chi$, and hence $\Delta \Theta$, is a steep function of $\alpha$ as well as $\beta$ (Figure 2), this approach can be very useful in constraining the viewing geometry.

\subsection{Demonstration using simulations}

To demonstrate how the modulation phase-offset would reflect in simultaneous multi-frequency observations, a carousel pattern similar to that shown in Figure 1(b) but with 6 sparks was simulated. The subbeams were assumed to originate from the sparks uniformly populated on a $90 \mathrm{~m}$ radius circular ring around the magnetic axis on the neutron star surface. The intensity distributions of the sub-beams were modelled as two-dimensional symmetric gaussian functions with halfpower widths nearly $25 \%$ of the carousel radius. To generate single pulse sequence at a desired frequency, the modulation sampled by the sightline at the corre- sponding emission altitude was mapped to the pulselongitudes. The pulse sequences at 240 and $610 \mathrm{MHz}$ were generated by assuming a carousel circulation period of $14.84 \mathrm{P}$ (and hence, a subpulse modulation period $\mathrm{P}_{3}$ of $2.47 \mathrm{P}$ ). Furthermore, normally distributed noise was added to the pulse-sequences such that the average signal-to-noise ratio $(\mathrm{S} / \mathrm{N})$ of resultant individual pulses was nearly 10 .

The different sampling of the carousel would result in the corresponding "drift-bands" at two frequencies to appear offset from each other. This offset in the subpulse modulation phase can be best viewed after folding the sequences modulo $\mathrm{P}_{3}$ (and hence increasing the significance of the drift-bands). The $\mathrm{P}_{3}$ modulo folded pulse sequences, hereafter called as $\mathrm{P}_{3}$-folds, are shown in Figure 3. The drift-bands or more generally, the modulation bands, i.e., the modulation patterns under the individual pulse-components, are clearly offset at two frequencies. The larger horizontal separation between the drift-bands at the lower frequency is due to RFM. The vertical offset between the corresponding modulation bands at the two frequencies is a consequence of slightly different sampling of the carousel at the polar cap. As expected, the vertical offsets are in opposite directions for the two components. By cross-correlating the modulation patterns at the peak positions of the leading component at the two frequencies (at about $-4.1^{\circ}$ and $-3.3^{\circ}$ of pulse-longitude), the offset is estimated to be about $0.11 \mathrm{P}_{3}$ (or $0.26 \mathrm{P}$ ). Note that this offset is primarily due to the first term in equation 3 , with the second and third terms contributing only about $0.8 \%$ and $0.1 \%$, respectively. An example of this effect on real data from PSR B0943+10 can be seen in figure 14 of Bilous (2018).

\subsection{Implications for determining carousel related physical parameters}

The pulse-to-pulse intensity fluctuation sequence corresponding to a particular component at a given frequency could be considered as a delayed or advanced version of that at a different frequency since the corresponding sightlines sample the underlying carousel at its different rotation phases. An expression equivalent to equation 3 can be written for the absolute delay between fluctua- 


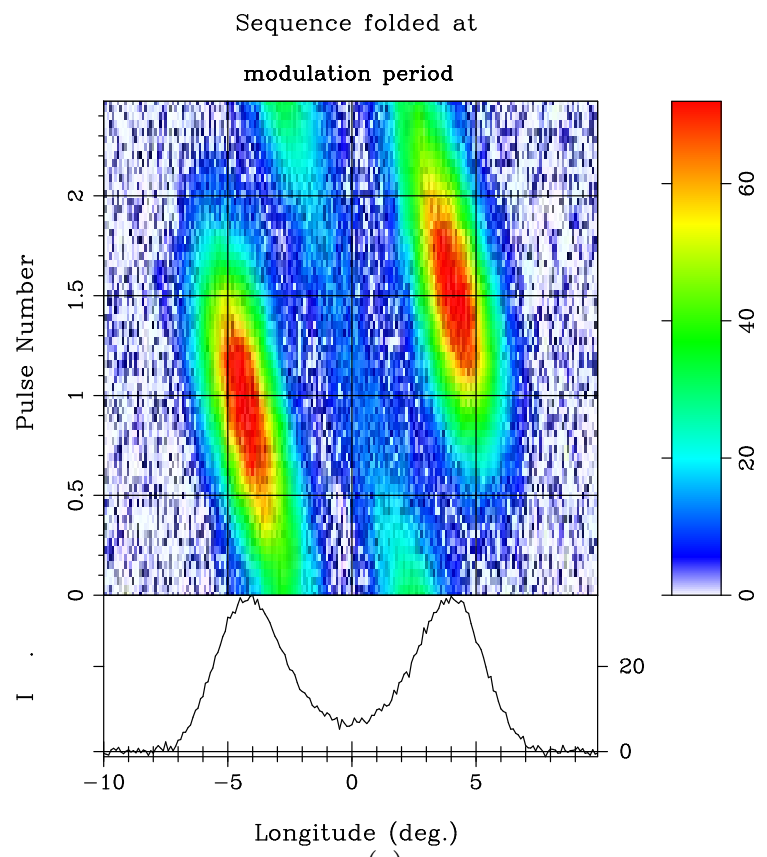

(a)

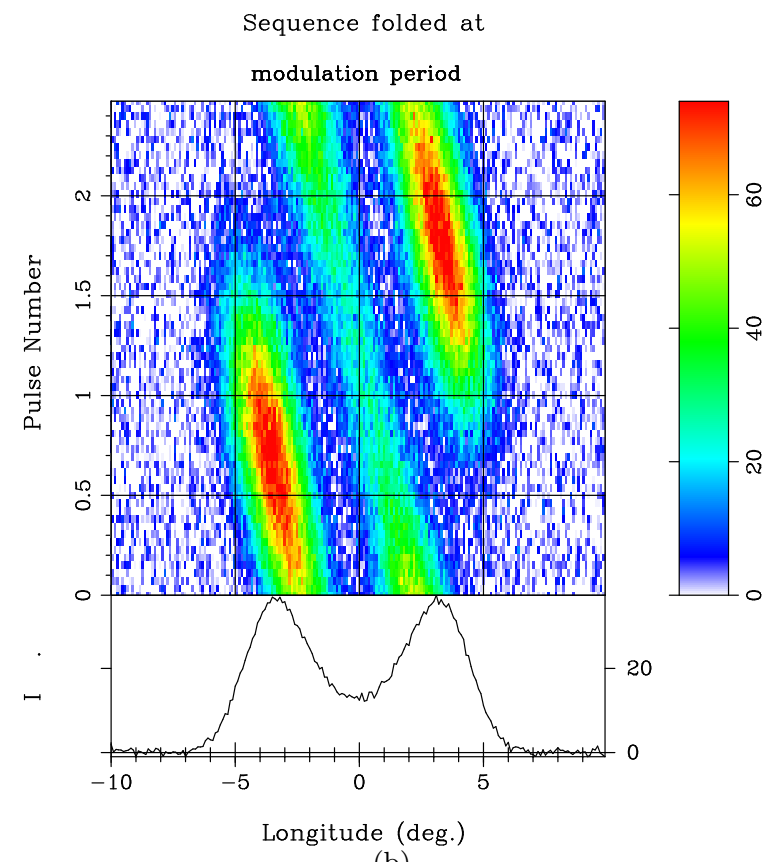

(b)

Figure 3. $\mathrm{P}_{3}$-folded modulation patterns for two pulse sequences simulated at $240 \mathrm{MHz}$ (left) and $610 \mathrm{MHz}$ (right). The change in separation of the two components is due to radius to frequency mapping. The vertical change in positions of the modulation bands under the corresponding pulse components is due to sampling of the carousel at slightly different rotation phases.

tions under a component at two frequencies. Considering only the first term in equation 3. a simplified expression for this delay, $\Delta t$, is:

$$
\Delta t=\frac{\Delta \chi}{\omega_{c}}
$$

where, $\omega_{c}$ is the angular velocity of the carousel ${ }^{2}$. Measurement of $\Delta t$ would provide direct estimates of the angular velocity of the carousel (and hence $\mathrm{P}_{4}$ ) and its circulation direction. Using the viewing geometry parameters and independently measured emission altitude, $\omega_{c}$ can be easily translated to the drift velocity of the sparks. Note that this method can provide a direct estimate of the the average drift velocity even if the underlying carousel is not stable enough to give rise to observable subpulse modulation. The drift velocity directly relates to the electric potential in the polar gap in the carousel model (Ruderman \& Sutherland 1975), and provides important feedback to the pulse emission models.

\subsubsection{Resolving the aliasing of $\mathrm{P}_{3}$}

The shortest $\mathrm{P}_{3}$ that can be measured without getting aliased is about twice the pulsar rotation period. An estimate of $\mathrm{P}_{4}$ in combination with the $P_{3}^{\text {obs }}$ can potentially constrain the aliasing. As shown by Deshpande \& Rankin (2001), solving for integer pairs of $\mathrm{n}$ and $N_{\mathrm{sp}}$ in $P_{4}=N_{\mathrm{sp}} /\left(n+P / P_{3}^{\text {obs }}\right)$ along with observed polarization constraints could provide the aliasing order. However, measuring $\mathrm{P}_{4}$ reliably and using it to resolve the $\mathrm{P}_{3}$-aliasing heavily depends on stability timescale of the carousel.

\footnotetext{
${ }^{2}$ For situations where the contributions from the second and third terms in equation 3 are significant, the corresponding terms should be incorporated in equation 4
}

The estimate of $\Delta t$ using simultaneous dual- or multifrequency observations enables an independent and efficient way of resolving the $\mathrm{P}_{3}$-aliasing. For a reasonably coherent subpulse modulation, the phase-offset in modulation at two frequencies under a specific component can be measured using, e.g., the $\mathrm{P}_{3}$-folds (similar to those presented in Figure 3). This measured phase-offset is related to the measured delay via:

$$
\Delta \Theta_{\text {obs }}=2 \pi\left(n+\frac{P}{P_{3}^{\text {obs }}}\right) \frac{\Delta t_{\text {obs }}}{P}
$$

where the subscript or superscript "obs" denotes that these are observational estimates. The estimates of $\Delta \Theta_{\text {obs }}, P_{3}^{\text {obs }}$ and $\Delta t_{\text {obs }}$ can be readily used to determine the permissible aliasing order. It is worth emphasizing here that equation 5 involves only observed quantities and does not depend on viewing geometry parameters. Moreover, this method is also independent of the shape or symmetry axis of the carousel, and can reliably be employed even for a partial carousel. A relevant demonstration can be found in Section 4.

\section{IMPLICATIONS FOR CAROUSEL RELATED OBSERVABLE PHENOMENA}

There are a variety of observed phenomena that are either considered to be generally associated with the carousel of sparks at the polar cap, or they are examined within the scope of the carousel model. The implications of the sightline sampling different parts of the carousel at different frequencies are discussed below in the context of some of these phenomena.

\subsection{Multi-altitude emission at same frequency}

Majority of pulsars exhibit average profiles with 5 or lesser number of components. These components are explained by the presence of a single or two nested hol- 
low emission cones centered at the magnetic axis and a 'core'-beam near the axis (Rankin 1983). However, the co-existence of two emission cones would indicate that of the corresponding presumably two concentric carousels in the polar cap region. Rankin (1993) suggested an interesting possibility that the emission apparent from the inner cone happens at a lower height along the same group of peripheral field lines that produce the outer cone emission components. In this "single frequency multialtitude emission" scenario, a single emission cone corresponding to a single carousel of sparks could produce multiple components in the observed profile.

Gangadhara \& Gupta (2001) measured the emission heights separately for different components of the bright pulsars PSR B0329+54 and proposed that the different components originate at different heights in the magnetosphere but along relatively nearby field lines. For PSR B1237+25, Maan \& Deshpande 2014, hereafter MD14) showed that the underlying emission patterns corresponding to the presumably inner and outer cones are significantly correlated with each other. They proposed that the same radio frequency emission in the two cones originates from a common seed pattern of sparks at two different altitudes. These two case studies have strengthened the hypothesis of multi-altitude emission in pulsars.

The multi-altitude emission scenario implies that components from presumed different emission cones sample the carousel at its multiple rotation phases even in a single frequency observation. Hence, as discussed in previous sections, the components from different cones should exhibit a modulation phase-offset in the multialtitude scenario. Moreover, the phase-offset is expected to have a sign reversal when examined for a pair of components originated on the other side of the fiducial plane, and provides an important test for the single frequency multi-altitude proposition. Furthermore, confirming the modulation phase-offsets and their opposite signs for the pairs on the two sides of the fiducial plane does not require any knowledge about the viewing geometry. For a known emission geometry, the measured phase-offsets can be used to measure carousel parameters as explained in Section 2.3. A detailed case study to test the multialtitude emission scenario is presented in Section 4 .

\subsection{Magnetic field twisting}

A progressive twist in the magnetic field geometry as we go in the relatively weaker field regions further away from the star has been speculated (Rankin et al. 2003, MD14). One of the ways to probe any twist in the field lines is to map the underlying emission patterns (in the form of a carousel of emission sub-beams) at different frequencies (i.e. at different altitudes) and examining any rotation of the pattern (Maan et al. 2013, MD14). While this method makes use of the underlying modulation and advantages of averaging, it suffers from most likely uneven distribution of modulation power in different components and could be misleading. Most of the pulsars have asymmetric profiles or with significantly different intensity under different pulse components. The underlying modulation power also varies significantly under different pulse components. The corresponding mapping of the emission patterns naturally gets dominant contributions from the components with stronger modulation powers. Along with the frequency or emission height dependent variation in modulation phase discussed above, the asymmetric or uneven distribution of modulation power could result in an apparent rotation of the mapped emission patterns even in the absence of any twist in the field geometry.

Examining the effects of field twisting on $\Delta \Theta$ would provide a cleaner probe. In the presence of a twist in field lines, equation 3 would get contribution from an additional term, $\delta \Theta_{\text {twist }}$, which would be a measure of the twist between the two emission altitudes under consideration. Importantly, $\delta \Theta_{\text {twist }}$ contribution will be in the same direction (i.e., without a sign reversal) on both sides of the fiducial plane. This aspect can be used to measure any twist in the magnetic field lines between two emission altitudes. If we denote the phase-offsets measured on the two sides of the fiducial plane as $\Delta \Theta_{1}$ and $\Delta \Theta_{2}$, then

$$
\delta \Theta_{\text {twist }}=\frac{\Delta \Theta_{1}+\Delta \Theta_{2}}{2}
$$

and the corresponding change in the magnetic azimuth of a field-line between the two emission heights can be expressed as $\delta \chi_{\text {twist }}$

$$
\delta \chi_{\text {twist }} \approx \frac{\delta \Theta_{\text {twist }}}{N_{s p}}
$$

\subsection{Pseudo-nulls observed at multiple frequencies}

Pseudo-nulls represent chance positioning of our sightline across the minima (i.e., in between the sub-beams) in the carousel (Herfindal \& Rankin 2007, 2009), as against the physical cessation of the emission in the actual nulls. Due to the above discussed frequency dependent phase-offset, pseudo-nulls can be expected to be non-simultaneous for carefully chosen observing frequencies. Since the actual nulls are generally broadband (Gajjar et al. 2014), the above aspect provides a critical test for the presence of pseudo-nulls.

\subsection{Wide-band observations are not necessarily more sensitive for single pulse modulation studies}

A pulse-sequence from a significantly wide-band observation would merge the resultant modulation from a finite angular width of the carousel. If multiple frequencies were to sample the same part of the carousel, RFM would have implied observing the same subpulse at different pulse-longitudes as the position of a pulse-component changes with frequency, which in turn, would imply the drift-bands to be wider in pulse-longitude for wide-band observations. However, as the corresponding subpulse modulation phase also changes with frequency, wideband observations imply the observed drift-bands to get vertically elongated. In case the corresponding change in pulse-width across the observed frequency range is smaller or comparable to the subpulse-width, the merging of subpulses at different rotation phase of the carousel implies that the subpulse modulation would get smeared, and might even be washed out for appropriate combinations of viewing geometry, $\mathrm{P}_{3}$, and observing bandwidth.

In addition to the frequency dependent phase-offset itself, its manifestation in some of the above discussed phenomena would provide critical tests of the carousel model. 

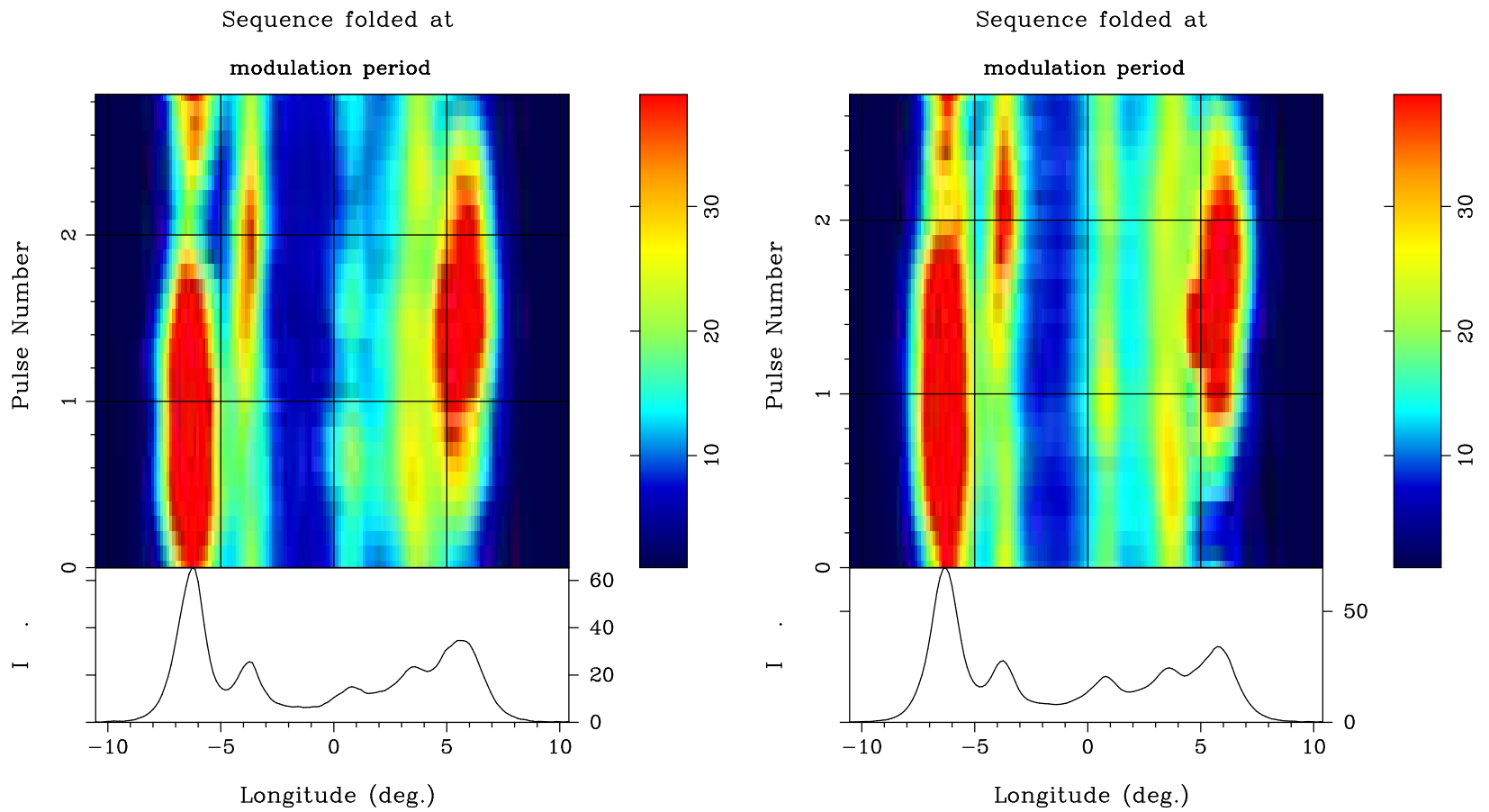

Figure 4. Left: The subsequence $S_{1}$ (pulse-number range 97-352) folded modulo $\mathrm{P}_{3}^{\text {obs }}=2.84 \mathrm{P}$. Right: The $\mathrm{P}_{3}$-folds for subsequence $S_{2}$ (pulse-number range 417-672) corresponding to it's $\mathrm{P}_{3}^{\text {obs }}$ of $2.72 \mathrm{P}$. The colors have been saturated a little to bring out the modulations clearly. Presence of modulation bands for all the four conal components, including component IV, are clearly visible for both the subsequences. The $\mathrm{P}_{3}$-folds were computed with reference to arbitrary starting pulses, however, the reference pulses were tried to be adjusted so as to get the patterns for the two subsequences nearly aligned for ease of comparison.

\section{MULTI-ALTITUDE EMISSION AND RESOLVING}

$P_{3}$-ALIASING: A CASE STUDY USING PSR B1237+25

The bright pulsar PSR B1237+25 exhibits a multicomponent profile resulting from a special viewing geometry and a rich variety of subpulse modulation in many of its components. The impact angle estimate $\left(|\beta| \lesssim 0.25^{\circ}\right.$; Srostlik \& Rankin 2005) indicates that our sightline traverses almost through the magnetic axis of this pulsar. The sightline presumably cuts through two hollow emission cones and a core beam giving rise to five components in the average profile (for example, see lower panels in Figure 4). For ease of reference in the discussion below, let us denote the five distinct components, starting from the leading most one as I, II, III, IV and V. Component I and $\mathrm{V}$ are the outer conal components, II and IV are the inner conal components, and III is the core component. The emission height estimates for the inner and outer cones using aberration-retardation considerations ( $\sim 280$ and $340 \mathrm{~km}$; Srostlik \& Rankin 2005) and those after taking care of even the sweep-back effects of the field lines ( $\sim 640$ and $715 \mathrm{~km}$; MD14), suggest the inner cone to be emitted at altitudes lower than the outer cone.

\subsection{Single frequency multi-altitude emission}

Various characteristics of B1237+25 were indeed some of the compelling reasons that Rankin (1993) proposed the multi-altitude emission hypothesis. Based on the correlation between the emission patterns corresponding to the two cones and the emission height estimates, MD14 provided a strong support to the multi-altitude scenario for this pulsar. Given that the outer conal components and at least one of the inner conal components exhibit rich variety of subpulse modulation (e.g., see Srostlik \& Rankin 2005, MD14), B1237+25 offers an interesting but challenging case to test the multi-altitude emission proposition by examining the modulation phase offsets in components corresponding to the two cones (cf. Section 3.1).

To test the multi-altitude emission scenario as well as to explore the scope of resolving the aliasing issue using methods proposed in Section 2.3, a detailed analysis of a 5209 rotation periods long pulse sequence from this star observed using the Arecibo telescope at $327 \mathrm{MHz}$ is presented here. The sequence has a time resolution equivalent to $0.133^{\circ}$ in pulse-longitude. More details of the observations as well as basic data reduction (e.g., dedispersion, Faraday rotation correction, etc.) can be found in Smith et al. (2013) and MD14 (sequence "A" therein).

As expected from a central sightline traverse, the pulseto-pulse intensity fluctuations in B1237+25 are primarily of the form of amplitude modulation with $\mathrm{P}_{3}^{\text {obs }}$ around 2.7-2.8 P. Moreover, the subpulse modulation does not seem to be stable over long durations, perhaps due to a temporally unstable carousel. This results in a broad frequency feature corresponding to $\mathrm{P}_{3}^{\text {obs }}$ (hereafter the $\mathrm{P}_{3^{-}}$ feature) in the longitude-resolved fluctuation spectrum (LRFS; Backer 1970c). To determine the modulation phase-offsets and test the multi-altitude emission proposition, all the four conal components need to exhibit the same modulation. However, noting from the previous studies, component IV hardly shows any indication of modulation. Presence of any faint modulation in this component is perhaps best sought in small sections of the sequence that could potentially exhibit a coherent modulation. To find instances of stable and coherent modulation at shorter timescales, a number of LRFS computed using 256-pulse wide blocks, with the starting of 
Table 1

Cross-correlation and phase-offsets between $\mathrm{P}_{3}$-folded modulation patterns.

\begin{tabular}{cccccc}
\hline \hline Subseq. & \multicolumn{2}{c}{ Components I and II } & & \multicolumn{2}{c}{ Components IV and V } \\
\cline { 2 - 3 } \cline { 5 - 6 } & $\rho_{\max }(\%)$ & $\Delta \Theta_{\text {obs }}\left({ }^{\circ}\right)$ & & $\rho_{\max }(\%)$ & $\Delta \Theta_{\text {obs }}\left({ }^{\circ}\right)$ \\
\hline$S_{1}$ & $91.0 \pm 6.7$ & $+137 \pm 32$ & & $76.5 \pm 16.4$ & $-104 \pm 36$ \\
$S_{2}$ & $95.6 \pm 3.4$ & $+137 \pm 22$ & & $71.3 \pm 19.0$ & $-115 \pm 47$
\end{tabular}

Note. $-\Delta \Theta_{\text {obs }}$ is the phase-offset by which the $\mathrm{P}_{3}$-folded modulation-patterns under the inner conal components II and IV lag behind those under the outer conal components I and $\mathrm{V}$, respectively, and it corresponds to the maximum normalized cross-correlation coefficient, $\rho_{\max }$.

successive blocks separated by 16 pulses, were examined. Two blocks, with the pulse-number ranges 97-352 and 417-672 (hereafter subsequence $S_{1}$ and $S_{2}$, respectively), were identified to have particularly strong and reasonably coherent $\mathrm{P}_{3}$-features. The corresponding $\mathrm{P}_{3}^{\text {obs }}$ in these two subsequences were measured to be $2.84 \mathrm{P}$ and $2.72 \mathrm{P}$, respectively. These subsequences folded modulo their respective $\mathrm{P}_{3}^{\text {obs }}$ are shown in Figure 4.

There are quite a few things to notice in the P3-folds presented in Figure 4. First of all, the coherency of the $\mathrm{P}_{3}$-feature in LRFS reflects in the form of clearly identifiable modulation bands for components I, II and V. Despite of having slightly different $\mathrm{P}_{3}^{\text {obs }}$ values, the overall modulation bands are similar for both the subsequences. The component IV generally does not show a distinct $\mathrm{P}_{3}$-feature in LRFS, but Figure 4 clearly shows that this component also exhibits modulation at the same $\mathrm{P}_{3}^{\text {obs }}$. The modulation band for component IV is less prominent than those for the other three components, perhaps because of a significant overlap with component $\mathrm{V}$ and since the modulations in these two components are clearly offset in phase. The modulation bands in components I and II are also offset, and interestingly, the offset appears to be similar as that between components IV and V but in opposite direction. Finally, the core component does not show much indication of a well defined modulation band.

To measure the modulation phase-offsets, the modulation patterns corresponding to the peaks of the inner and outer conal components (after averaging over a few bins around the peak) were extracted from the $\mathrm{P}_{3}$-folds and cross-correlated with each other. Measurements of the maximum normalized cross-correlation coefficients as well as the corresponding phase-offsets $\left(\Delta \Theta_{\text {obs }}\right)$ are given in Table 1. Note that the measured correlation between the modulation patterns is highly significant. The larger uncertainties for the pair of components IV and $\mathrm{V}$ are perhaps due to the significant overlap between the two components. Modulation in component II lags behind that in I, while the modulation in component IV leads that in $\mathrm{V}$ by similar amounts within the measurement uncertainties. It was also confirmed that the modulation patterns, and hence the phase-offset measurements, are not affected by any profile or polarization mode changes (see Appendix).

The above measurements clearly show that the phaseoffsets in modulations under the inner and outer conal component pairs on the two sides of the fiducial plane are in agreement with the expectations from a multi-altitude emission scenario. Noting again that (i) the inner cone is emitted at a lower altitude than the outer cone, (ii) the modulations in the inner and outer conal components are phase-locked and exhibit same $\mathrm{P}_{3}$, and (iii) the phaseoffsets in these corresponding modulations are equal and opposite on the two sides of the fiducial plane, it seems almost inevitable to conclude that multi-altitude emission along nearby bunches of field lines seeded by a single carousel of sparks in the polar cap gives rise to the observed average and modulation properties of B1237+25.

The "modified carousel model" (Gil \& Sendyk 2000) proposes maximal packing of the sparks on the polar cap and can explain the subpulse modulation in multiple cones originating from multiple carousels. Due to a characteristic spark dimension that is similar to separation between consecutive sparks, this model predicts the number of sparks in the inner carousel to be less than those in the outer carousel. However, at least for two doublecone pulsars B0818-41 (Bhattacharvva et al. 2009) and $\mathrm{B} 1237+25$ (MD14), the number of subbeams in the two apparent carousel rings have been found to be same. Hence, the multi-altitude emission is perhaps the only viable explanation for the observed properties in doublecone pulsars. Given the similar observed properties, including the phase-locked modulation (also see detailed discussion in MD14), for many other multiple component pulsars, the above modulation phase analysis could be used to confirm the multi-altitude emission to be, perhaps, a common phenomena in pulsars.

MD14 also observed magnetic azimuth offsets between the carousel patterns mapped for the inner and outer conal component pairs. They associated this offset to a potential twist in the emission columns (and hence the magnetic field geometry) between the two different emission altitudes. However, as apparent from their figure 2 , the subpulse modulation power under component II heavily dominates that under component IV (it is also apparent from Figures 4 and 5 in this work). Hence, as explained in Section 3.2. the analysis in MD14 to determine any twist could have been affected by the highly uneven distribution of modulation powers under the two inner conal components. If that is indeed the case, the magnetic azimuth shift measured in MD14 multiplied by the corresponding number of sparks would be a quantity directly comparable to $\Delta \Theta_{o b s}$ in this work. Using their tables 1 and 2 , this quantity is $180^{\circ} \pm 25^{\circ}, 156^{\circ} \pm 42^{\circ}$, $117^{\circ} \pm 45^{\circ}$ and $80^{\circ} \pm 36^{\circ}$, corresponding to the four subsequences presented therein, and is largely consistent with $\Delta \Theta_{\text {obs }}$ within the measurement uncertainties (Table 1 ). Hence, the magnetic azimuth offsets measured in MD14 could more likely be explained as the modulation-phase offsets between components I and $\mathrm{II}^{3}$ - a natural consequence of multi-altitude emission and the carousel model rather than a twist in the emission columns. However, the measurements in Table 1 might give an impression of small difference in the phase-offsets on the two sides of the fiducial plane, which would hint towards a small twist $\left(\delta \chi_{\text {twist }}=\left[16^{\circ} \pm 24^{\circ}\right] / N_{s p}\right.$; much smaller than that in MD14 for number of sparks more than a few) in the field lines (Section 2.3). But within the current measurement uncertainties, this analysis does not present an evidence

\footnotetext{
3 In carousel mapping, the modulations in the outer conal components $\mathrm{I}$ and $\mathrm{V}$ would get added in phase, and the modulation power in II dominates that in IV.
} 
Table 2

Absolute delays between pulse-to-pulse intensity fluctuations under different components.

\begin{tabular}{cccccc}
\hline \hline \multirow{2}{*}{ Subseq. } & \multicolumn{2}{c}{ Components I and II } & & \multicolumn{2}{c}{ Components IV and $\mathrm{V}^{\dagger}$} \\
\cline { 2 - 3 } \cline { 5 - 5 } & $\Delta t_{\text {obs }}(\mathrm{P})$ & $\Delta \Theta_{\text {imp }}\left({ }^{\circ}\right)$ & & $\Delta t_{\text {obs }}(\mathrm{P})$ & $\Delta \Theta_{\text {imp }}\left(^{\circ}\right)$ \\
\hline$S_{1}$ & $+1.09 \pm 0.14$ & $+137 \pm 18$ & & $-0.55 \pm 0.40$ & $-68 \pm 50$ \\
$S_{2}{ }^{\ddagger}$ & $+1.30 \pm 0.35$ & $+173 \pm 47$ & & $-0.52 \pm 0.50$ & $-68 \pm 65$
\end{tabular}

Note. - The delay $\left(\Delta t_{\text {obs }}\right)$ measurements indicate how much the pulse-to-pulse intensity fluctuations under the inner conal components II and IV lag behind those under the outer conal components I and $\mathrm{V}$, respectively. $\Delta \Theta_{\mathrm{imp}}$ is the phase-offset implied by the measured delay, i.e., $\Delta \Theta_{\mathrm{imp}}=360^{\circ} \times \Delta t_{\mathrm{obs}} / \mathrm{P}_{3}$, assuming the aliasing order to be 0 .

$\dagger$ The significant overlap between the components IV and V makes it harder to measure the underlying phase-gradient. Although a definitive phase-gradient and corresponding delay has been measured, it is possible that the overlap of the two modulations has contaminated the measurements, see text for more discussion.

$¥$ These measurements have utilized only first 100 pulses in subsequence $S_{2}$ to avoid the contamination due to abnormal mode instance.

supporting the field twist. A more detailed analysis using lower frequency observations, where the outer and inner conal components, particularly components IV and V, would perhaps be better separated, is under progress.

\section{2. $\mathrm{P}_{3}$-aliasing and carousel parameters}

With the above supporting evidence for multi-altitude emission, the pulse-to-pulse intensity fluctuations under the outer conal components can be viewed as delayed versions of those under the corresponding inner conal components. The delay, if measurable, can be used to resolve the $\mathrm{P}_{3}$-aliasing as discussed in Section 2.3. In principle, the single pulse intensity fluctuations corresponding to different components can be cross-correlated to estimate any underlying delay. However, such an approach would be severely limited by our sampling of the fluctuations only once every rotation period. Instead, we can compute the cross-spectrum of two fluctuation sequences of interest and look for any significant gradient in the crossspectrum phases. This technique can be used to measure any fractional delays with better precision, and is often used in antenna and digital signal processing. Using this method, the measurements of fractional delays $\left(\Delta t_{\text {obs }}\right)$ between fluctuation sequences under the above discussed pairs of components are presented in Table 2 .

As evident from Table 2 the delay between fluctuations under components I and II is highly commensurate with the corresponding phase-offset measurements in Table 1 . The delay measurements for the component pair IV and V are also consistent with the corresponding phase offsets within the uncertainties, though there is a hint that the delays are underestimated. The modulations in the components IV and V are offset by more than a quarter of $\mathrm{P}_{3}$-cycle, and their significant overlap is prone to contaminate the resultant phase gradient in the cross-spectrum. Since the underlying fluctuation spectrum is same, the overlap of two out-of-phase modulations under these two components would result in underestimation of the actual phase gradient, and hence that of the corresponding delay ${ }^{4}$. Due to these reason, below we use the delay estimates only for the components I and II to determine the aliasing order.

Using the $\Delta \Theta_{o b s}$ and $\Delta t_{o b s}$ measurements for the pair of components I and II (Tables 1 and 2) in equation 5 results in the aliasing order estimates of $0.0 \pm 0.09$ and $-0.08 \pm 0.09$ for the subsequences $S_{1}$ and $S_{2}$, respectively. Since $n$ has to be an integer, the only permissible solution is 0 . Note that the immediate next aliasing orders $(|n|=1)$ are highly inconsistent with the above estimates. Hence, within the multi-altitude emission scenario, it is fairly evident that $\mathrm{P}_{3}^{\text {obs }}$ of $2.7-2.8 \mathrm{P}$ is the actual $\mathrm{P}_{3}$.

Now equation 3 can also be used to estimate the number of sparks. Since the $\Delta \Theta_{o b s}$ estimates for the component pair IV and V may be affected by their overlap, only the corresponding estimates between the first two components will be used below. To compute $\Delta \phi$ and $\Delta \chi$, first the positions of the components are needed. Srostlik \& Rankin (2005) provide (in their table 1) the positions of the conal components as well as the aberration-retardation longitude-shifts ${ }^{5}$. After correcting for the aberration-retardation shifts, the positions of the components I and II are $-6.03^{\circ} \pm 0.22^{\circ}$ and $-3.66^{\circ} \pm 0.13^{\circ}$, and hence $\Delta \phi=2.37^{\circ} \pm 0.26^{\circ}$. Using $n=0$, the second term in equation 3 contributes only about $0.6 \%$ of the observed $\Delta \Theta$ of $137^{\circ}$ (Table 1). The contribution from the third term is expected to be even smaller. Using the above component positions in equation 1, $\Delta \chi$ is estimated to be $1.52^{\circ} \pm 0.25^{\circ}$ for $\alpha=57.6^{\circ}$ and $\beta=-0.3^{\circ}$ (Smith et al. 2013). Equation 3 then suggests the number of sparks to be some $60-133$. If we also include an uncertainty of $\pm 0.1^{\circ}$ in $\beta$, this estimate becomes even less constraining to 40-240. The corresponding estimate for the carousel circulation period would be in the range $113-680 \mathrm{P}$. Using $\Delta t_{o b s}$ estimates in equation 4 also provides similar estimates. Better constraints on the number of sparks as well as $\mathrm{P}_{4}$ will require more precise estimates of $\Delta \Theta_{o b s}$ and $\beta$.

\section{CONCLUSIONS}

Simultaneous multi-frequency observations have been utilized to probe and characterize several properties of pulsars. However, observable geometric effects of radiusto-frequency mapping in the subpulse modulations manifested by the underlying carousel of sparks have largely remained unexplored. This work presents the conceptual as well as mathematical formulation of the expected geometric imprints of the carousel in simultaneous multifrequency or wideband observations. The main conclusions of this work are summarized as follows.

1. A formulation has been presented for the expected change in modulation phase under a pulse component observed simultaneously at two or more frequencies. The phase-offset is illustrated using simulated pulse sequences at two frequencies, and it's

${ }^{4}$ Note that, in this situation, the cross-correlation of the corresponding $\mathrm{P}_{3}$-fold patterns (as in Table 1) would still provide less contaminated estimates of $\Delta \Theta$ as long as the intrinsic modulation powers in the individual components dominate the corresponding modulation patterns.

${ }^{5}$ Independently estimated positions by fitting the average profile using a number of Gaussian functions were found to be consistent with those from Srostlik \& Rankin (2005). 

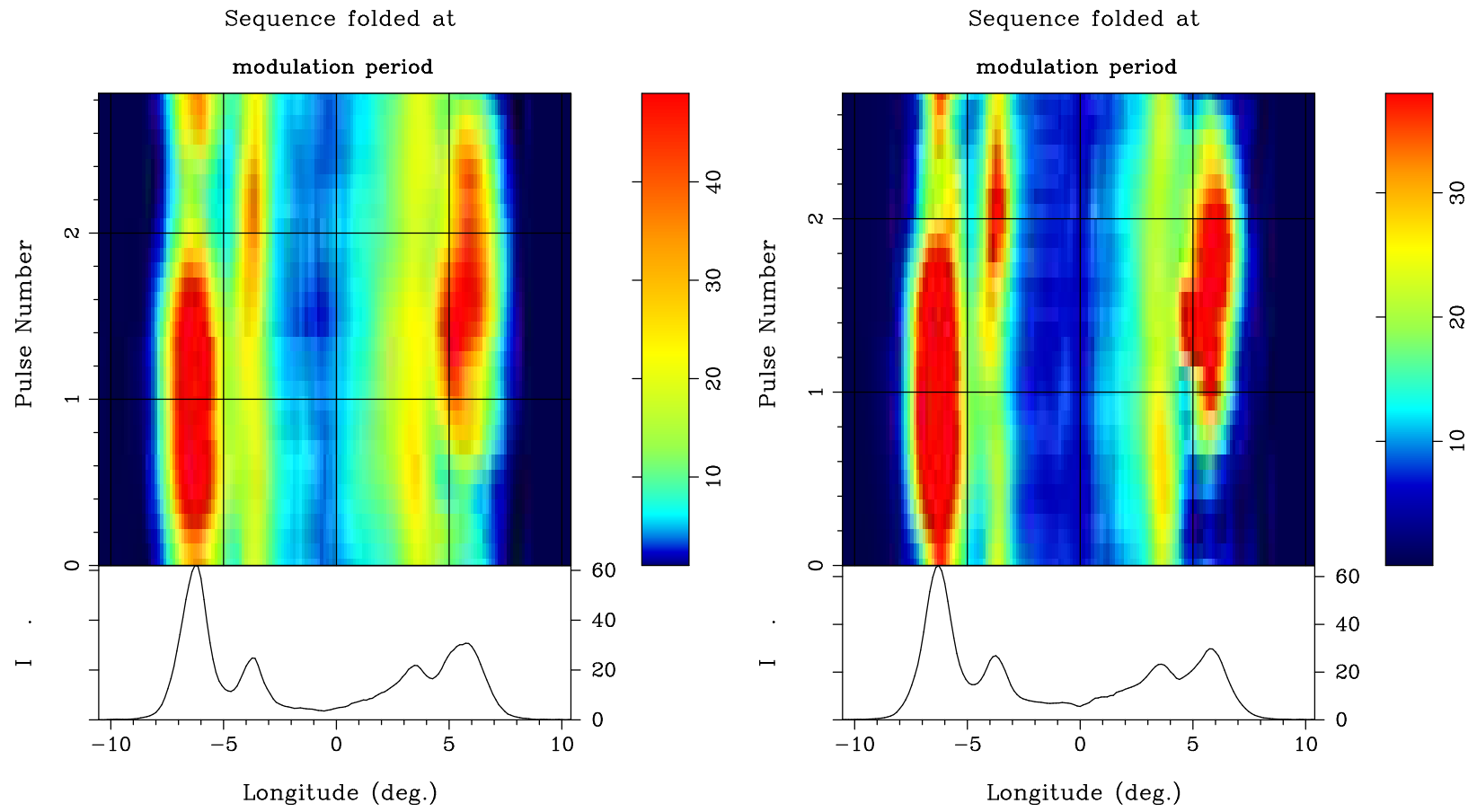

Figure 5. Same as in Figure 4 but for the PPM powers from the subsequence $S_{1}$ (left) and $S_{2}$ (right).

expected variations with the viewing geometry are demonstrated with a reasonable assumption about the frequency dependence of the emission cone size.

2. It is shown that the above phase-offset can be used to resolve the aliasing issue in the measured $\mathrm{P}_{3}$ without relying on knowledge of the viewing geometry of the pulsar. For a known emission geometry, the phase-offset can also be used to determine various physical parameters associated with the carousel, e.g., the average velocity of the sparks and the carousel circulation period.

3. The phase-offset between subpulse modulations observed simultaneously at two or more frequencies has important implications in probing several phenomena (e.g., single frequency multi-altitude emission, field twisting, orthogonal polarization modes emission sites, etc.) as well as scrutinizing the carousel model and related phenomena (e.g., the pseudo-nulls).

4. A detailed analysis of a $327 \mathrm{MHz}$ pulse sequence from B1237+25 has been presented uncovering the modulation even under the inner conal component on the trailing side. The phase-offsets between modulations under the inner and outer conal components are presented as a strong evi- dence for single-frequency multi-altitude emission in $\mathrm{B} 1237+25$. Within this multi-altitude scenario, it is shown that the measured $\mathrm{P}_{3}$ of $2.7-2.8 \mathrm{P}$ is definitely not aliased.

The formulation, methods and analysis presented here can be readily applied to already existing as well as future simultaneous multi-frequency or wideband observations to scrutinize pulsar emission models and provide further observational feedback.

I sincerely thank the anonymous referee for constructive comments which helped in improving the manuscript significantly. I am grateful to Joanna Rankin for making available the $327 \mathrm{MHz}$ pulse sequence discussed in Section 4 as well as its polarization mode separated version used in the Appendix. I also sincerely thank Joeri van Leeuwen and Andrzej Szary for their valuable feedback on the manuscript. A part of the work presented here made use of a modified version of the single pulse analysis software SPULSES, which was originally developed by Prof. Avinash A. Deshpande at the Raman Research Institute, Bangalore, India. I acknowledge use of the funding from the European Research Council under the European Union's Seventh Framework Programme (FP/2007-2013)/ERC Grant Agreement no. 617199.

\section{APPENDIX}

Like several other pulsars, B1237+25 exhibits two modes: normal and abnormal modes (Backer 1970a, b, 1973), which are marked by significantly different profile shapes as well as the relative orthogonal polarization mode (hereafter OPM) powers. The classical normal mode was later found to consist of two different sub-modes: a flare-normal mode defined by bright core activity and a quiet-normal mode with little or no core activity (Srostlik \& Rankin 2005). The subpulse modulation properties could differ in different modes. However, we note that there is no noticeable instance of abnormal mode pulses in subsequence $S_{1}$, while subsequence $S_{2}$ shows one transition to the abnormal mode for about 20 periods. By examining the $\mathrm{P}_{3}$-folds using only the normal mode pulses before and after the abnormal mode instance, it was confirmed that the $\mathrm{P}_{3}$-folds presented in Figure 4 are not affected by the mode-change in subsequence $S_{2}$. As 
proposed by Rankin \& Ramachandran (2003), the emission elements giving rise to the modulating powers in the two OPMs, namely the primary polarization mode (PPM) and the secondary polarization mode (SPM), could also have geometrical offsets. Such offsets, if any, could smear the modulation bands in Figure 4 . To examine the possible presence of any such effects, a two-way polarization separated version of the pulse sequence was used to obtain the $\mathrm{P}_{3}$-folds for the two subsequences using only the PPM power. These $\mathrm{P}_{3}$-folds are presented in Figure 5 and clearly show that the modulation bands in the conal components are identical to those obtained using the total power in Figure 4. The core-component mostly consist of SPM power, and hence, it is nearly absent in Figure 5. If at all, the near absence of core component in Figure 5 makes it further easier to notice the phase-offset between the modulations under component IV and V.

\section{REFERENCES}

Asgekar, A., \& Deshpande, A. A. 2001, MNRAS, 326, 1249

-. 2005, MNRAS, 357, 1105

Backer, D. C. 1970a, Nature, 228, 42

Backer, D. C. 1970b, Nature, 228, 752

Backer, D. C. 1970c, Nature, 228, 1297

Backer, D. C. 1973, ApJ, 182, 245

Backus, I., Mitra, D., \& Rankin, J. M. 2010, MNRAS, 404, 30

Bhattacharyya, B., Gupta, Y., \& Gil, J. 2009, MNRAS, 398, 1435

Bilous, A. V. 2018, A\&A, 616, A119

Blaskiewicz, M., Cordes, J. M., \& Wasserman, I. 1991, ApJ, 370, 643

Cordes, J. M. 1978, ApJ, 222, 1006

Deshpande, A. A., \& Rankin, J. M. 1999, ApJ, 524, 1008

一. 2001, MNRAS, 322, 438

Drake, F. D., \& Craft, H. D. 1968, Nature, 220, 231

Lorimer, D. R., \& Kramer, M. 2004, Handbook of pulsar astronomy

Dyks, J., Rudak, B., \& Harding, A. K. 2004, ApJ, 607, 939

Edwards, R. T., \& Stappers, B. W. 2002, A\&A, 393, 733

-. 2003, A\&A, 410, 961

Edwards, R. T., Stappers, B. W., \& van Leeuwen, A. G. J. 2003, A\&A, 402, 321

Gajjar, V., Joshi, B. C., Kramer, M., Karuppusamy, R., \& Smits, R. 2014, ApJ, 797, 18
Gangadhara, R. T., \& Gupta, Y. 2001, ApJ, 555, 31

Gupta, Y., \& Gangadhara, R. T. 2003, ApJ, 584, 418

Gil, J. A., \& Sendyk, M. 2000, ApJ, 541, 351

Herfindal, J. L., \& Rankin, J. M. 2007, MNRAS, 380, 430

-. 2009, MNRAS, 393, 1391

Kijak, J., \& Gil, J. 2003, 397, 969

Maan, Y. 2018, Pulsar Astrophysics the Next Fifty Years, 337, 366

Maan, Y., \& Deshpande, A. A. 2014, ApJ, 792, 130

Maan, Y., Deshpande, A. A., Chandrashekar, V., et al. 2013, ApJS, 204, 12

Mitra, D., \& Rankin, J. M. 2008, MNRAS, 385, 606

Rankin, J. M. 1983, ApJ, 274, 333

-. 1993, ApJ

Rankin, J. M., \& Ramachandran, R. 2003, ApJ, 590, 411

Rankin, J. M., Suleymanova, S. A., \& Deshpande, A. A. 2003, MNRAS, 340, 1076

Ruderman, M. A., \& Sutherland, P. G. 1975, ApJ, 196, 51

Smith, E., Rankin, J., \& Mitra, D. 2013, MNRAS, 435, 1984

Srostlik, Z., \& Rankin, J. M. 2005, MNRAS, 362, 1121

Szary, A., \& van Leeuwen, J. 2017, ApJ, 845, 95

van Leeuwen, J., \& Timokhin, A. N. 2012, ApJ, 752, 155 